\title{
Boccia Court Analysis for Promoting Elderly Physical Activity
}

\author{
Alexandre Calado ${ }^{1}$, Pedro Leite ${ }^{1}$, Filomena Soares ${ }^{1}$, Paulo Novais ${ }^{1}$, and Pedro Are- \\ zes $^{1}$ \\ ${ }^{1}$ Algoritmi Centre, University of Minho, Guimarães, Portugal \\ alexandreluiscalado@gmail.com, a66161@alunos.uminho.pt, \\ fsoares@dei.uminho.pt, pjon@di.uminho.pt, parezes@dps.uminho.pt
}

\begin{abstract}
Physical inactivity is one of the leading risk factors for global mortality. Older adults, in particular, are more probable to suffer the consequences of physical inactivity, since it is one of the most sedentary age groups. On the other hand, engaging physical activity can have various benefits for the prevention of several diseases and functional loss prevention, therefore, it is critical to encourage its regular practice amongst the elderly. Boccia is a simple precision ball sport that is easily adaptable for individuals with physical limitations, which makes it a perfectly good game for this circumstance. The present paper proposes a ball-detection based system for monitoring the Boccia court, compute the current game score and display it on a user interface. The future goal of such system will be to motivate the elders to participate more frequently in the Boccia game and make the overall game experience more enjoyable. The proposed system was tested with twenty video recordings of different simulated game situations. Overall, the obtained results were encouraging, having only one incorrect game score being computed by the developed algorithm.
\end{abstract}

Keywords: Boccia, Object Detection, Sports Analysis, Physical Inactivity

\section{$1 \quad$ Introduction}

According to the World Health Organization [1], physical inactivity can be defined as an absence of bodily movement produced by skeletal muscles that require energy expenditure. It has been identified as the fourth leading risk factor for global mortality, which represents $6 \%$ of deaths globally and it is also associated with increased risk of type 2 diabetes mellitus, cardiovascular diseases, obesity, breast and colon cancers and other chronic diseases $[1,2]$.

Matthews et al. [3] identified older adults (aged $\geq 60$ years) to be the most sedentary group in the United States. It has been shown in previous studies that this age group can have substantial benefits from the practice of physical activity such as preventing functional loss [4], reducing the risk of falling [5], controlling blood pressure [6], improving bones and joint health [2] and even maintaining mental health [7]. However, $50 \%$ of sedentary adults have no intention of starting an exercise plan and from those who are engaged in physical activity, only $30 \%$ of older man and $15 \%$ of older woman

adfa, p. 1, 2011.

(C) Springer-Verlag Berlin Heidelberg 2011 
perform it regularly [8]. Facing these statistics, along with the current increase in older population, it is paramount to find innovative solutions for encouraging the elderly to engage in physical activity on a regular basis.

Boccia is a simple precision ball sport that resembles pétanque. For the context of this paper, it has the main advantage of being easily adaptable according to the age and limitations of the players. Besides, Boccia can be played as a team sport which promotes social interaction and encourages the individual to participate more often.

This paper proposes a system that monitors the Boccia game scenario through the use of a camera and, by computing an algorithm based on object detection, it returns the game score, in real time, to a User Interface (UI) that can be consulted by all of the players during the game.

The proposed system will be used during a Boccia game in a nursing home environment with the objective of making the game experience more enjoyable for the player and promoting the Boccia practice amongst the elderly.

In a later stage of this project, this system will be used synchronously with the recording of acceleration data of the player's arm, along with the Kinect to determine angles and analyse movements during the game $[9,10]$. Afterwards, all of the extracted data will be automatically processed to help enhance the player's performance by suggesting improvements in the movement of the ball. This data will also be available to the caregiver to detect physical or cognitive declines in the individual.

The paper is structured as follows: in section 2 a state-of-art about object detection and tracking applied to sports is presented, along with a brief description of Boccia and the respective scoring rules. Section 3 presents the proposed system's architecture. Section 4 described the used methodology to test the proposed system and respective results. Finally, in section 5, conclusions and future work are addressed.

\section{Background}

To the best of the authors' knowledge, there are no studies referencing Boccia as a context for object detection or tracking, nonetheless, this type of techniques has been applied with success to several other sports.

For instance, [11] used six monochrome cameras to track a tennis ball in 3D, based on its motion, intensity and shape. The system was successfully used during the international television broadcasts of tennis matches and provided the enabling of virtual replays, game statistics and other interesting features. Basketball has also been a target of various studies associated with ball tracking. Wu et al. [12] successfully tested a ball tracking algorithm on videos from a basketball tournament. This algorithm consisted in detecting the ball based on its colour and shape and, if the ball was indeed detected, the actual tracking would start in the following frame. Chen et al. [13] proposed a more complex approach by applying a physics-based algorithm for predicting the trajectory of a basketball in 3D. The algorithm was similar to the one developed by [12], however, it exploited the 2D shooting trajectory, along with the detection of the court lines, to reconstruct the 3D ball trajectory and infer the shooting location, which can be very 
useful in the context of game analysis. An analogous approach was developed for volleyball [14].

Curling, similarly to Boccia, is a strategy-based sport that depends of the curling stone's position, therefore, it is relevant to automatically annotate it by using object tracking algorithms, as observed in [15]. In this work, mean-shifting tracking was applied by using the detection of colour and edges of the curling stone. In certain situations of the game, the stone may become occluded by one of the players, which can lead to tracking interruption. To overcome this problem, when the curling stone becomes occluded, the algorithm maintains the tracking by using Kalman prediction [16].

Overall, the use of object detection and tracking applied to sports can provide the spectator with innovative ways of experiencing sport, such as highlighting important events, along with the gathering of richer data for statistical analysis. It can also provide valuable assistance for training, through tactics analysis, and relevant information for referee decision [17].

\subsection{The Boccia Game}

Boccia is a ball precision game that became a Paralympic sport in 1984. It can be played individually or by teams, which will be the focus of this paper. The game is played with one white ball, which is called the jack, along with six blue and six red balls. Each of the six different coloured balls is given to a team composed by three players, the red or blue team, respectively. Since the main feature of the proposed algorithm is returning the game score in real time, this section will focus on the Boccia rules regarding scoring.

The game is divided into six segments, which are called "ends". Throughout each end, each team throws their respective six balls as close as possible to the jack. As each end finishes, the score is annotated as it follows:

- The team that placed the ball closer to the jack will earn one point for each ball placed at a shorter distance from the jack than the closest opponent's ball to the jack.

- If two or more balls of different colour are equidistant from the jack, then each team earns a point per ball.

After all the six ends have been played, the points from each end are summed and the team that finished with a higher number of points is declared the winner. In case of a tie, extra ends will be played and the first team to win one will be considered the winner. The interested reader should refer to [18] for further knowledge about the current international Boccia rules.

For promoting physical activity, Boccia features the main advantage of being easily adaptable according to limitations and age of the player. The rules and size of the field can also be readapted for a nursing home, which makes Boccia a very accessible sport for older adults [19]. 


\section{Proposed System}

\subsection{Architecture}

The proposed system (Figure 1) relies on three main components: object detection device, processing unit and interface.

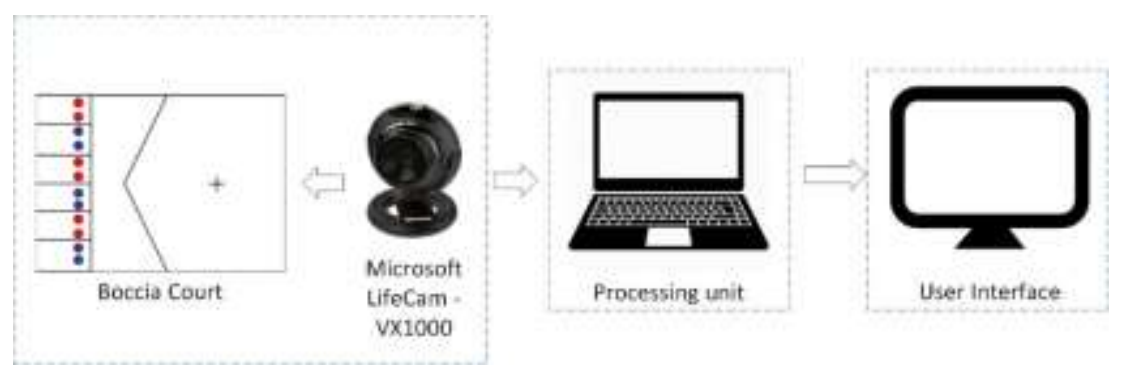

Fig. 1. System Architecture.

The selected device was a Microsoft LifeCam VX-1000 computer webcam, which provides a video stream, in real-time, to be processed by the algorithm in the computer, which was developed using Python programming language. In each of the video stream frames, the algorithm automatically detects the balls included in the camera's field-ofview (FOV) according to their colour: white, red or blue. After the white ball is detected, the distances between any blue or red ball covered by the FOV and the jack are calculated in pixels by using the centroid coordinates of each of the contours segmenting each ball, which are also calculated in pixels. Based on these values, the algorithm computes the score of the current game scenario according to the Boccia rules.

Finally, the connection between the algorithm and the UI is enabled by using TCP/IP communication. Each second, the current game score is sent by the algorithm to the UI which is displayed on a screen.

\section{$4 \quad$ Preliminary Results}

The proposed system was tested in a nursing home in S. Torcato, Guimarães, in Portugal, in a wide room that was used by the elders to play Boccia with adapted rules. As referenced by the caretaker, every Boccia game would start with the jack placed at an arbitrary distance from the players, depending on the individual's limitations. Furthermore, no court lines are considered during gameplay, and players throw the Boccia balls while sitting on a chair.

Since the system is based on object detection, it was preponderant to position the camera facing the court in a way that the limited 2D perspective would not interfere with the distance calculation between each Boccia ball and the jack.

Another important factor to have into account while positioning the camera is the luminosity. The level of luminosity and light angle have a direct effect on how the algorithm detects colours, which can lead to false positives or even the detection of 
incorrect colours. Considering the player's perspective when facing the court, there was a window with a curtain on the right side and lights on the ceiling, directly above the court. The authors considered these to be the elements that could be adjusted for optimizing the luminosity level. After testing the system under different light conditions, it was decided that the testing should be performed with the ceiling lights on and the window curtain completely closed. Again, considering the player's perspective facing the court, the system was tested using two camera locations:

- Camera 1: placed on left side of the court, facing the window, at a height of approximately $2,15 \mathrm{~m}$ (using a table and a tripod);

- Camera 2: placed on the right side of the court, facing the left side wall, at a height of approximately $2,12 \mathrm{~m}$ (using a table and a tripod);

For each of the camera locations, ten game situations depicting different game scores were simulated using the jack and just three blue balls and three red balls. Each of these game situations was recorded for 20 seconds and all the Boccia balls were positioned at an arbitrary distance from the jack and included in the camera's FOV before each recording. After all the recordings were performed, the algorithm parameters were adjusted to optimize colour detection and eliminate false positives. Finally, the algorithm was tested in each of the recordings and the computed game score was compared to the real game score for each situation.

Overall, the obtained results were encouraging. Considering position 1, the algorithm computed an incorrect game score for only one of the ten recordings. On the other hand, for position 2, all of the ten computed game scores were correct. Figure 2 depicts one of the game situations tested by the algorithm plus the corresponding game score and the current end being played, both displayed in the UI. There is a table on each side of the UI that keeps track of the points scored in the end of each of the six ends by the blue and red team, respectively. The total score of the respective team is shown at the bottom of each of these tables. Finally, in the bottom center of the UI, there are two buttons. The left button should be clicked on when each end finishes to store the score in the table. Moreover, the right button should be clicked on when the players desire to restart the game, which will reset all the points stored in the score tables to zero.
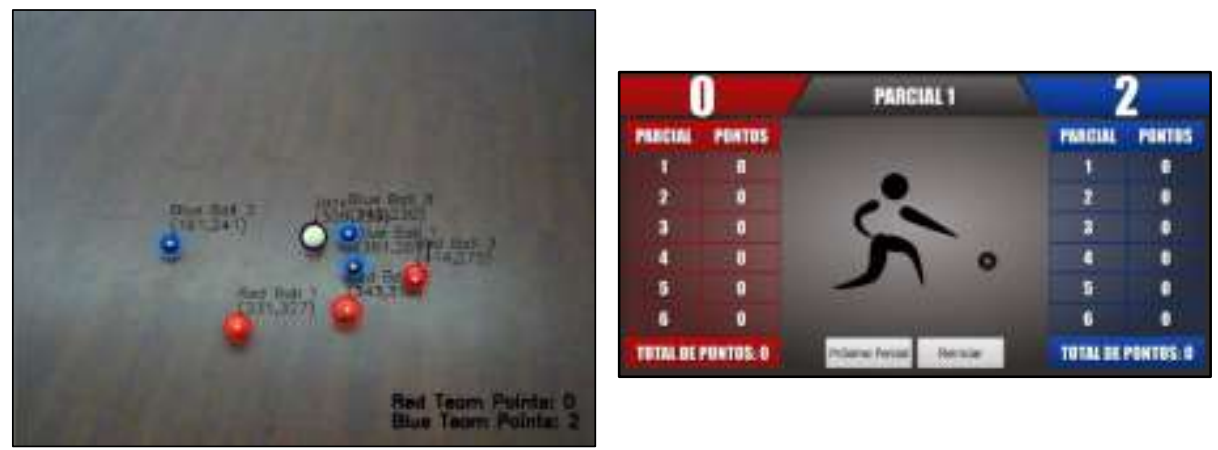

Fig. 2. At the left: Example of one of the recorded game situations, using camera position 2. At the right: The UI, in Portuguese, showing the corresponding game score of the current end ("parcial") being played. 


\section{$5 \quad$ Final Remarks}

The present paper proposed a system based on a ball detection algorithm to monitor the court of a Boccia game and automatically compute the game score to be displayed in a UI. The objective of such system is to motivate the elderly to engage more frequently in the game of Boccia and therefore promoting physical activity.

The system was tested by using the developed algorithm on recordings of Boccia game situations simulated in a space that belonged to a nursing home, where the elders usually played the game with adapted rules. Due to luminosity conditions, videos were recorded by using two distinct locations of the camera.

Considering the total of twenty recorded videos, the algorithm returned an incorrect score for only one of the game situations recorded with camera in position 1 . This error might have been due to the non-optimal camera location, which could have lead the limited 2D perspective to interfere with the computation of the distance between the Boccia balls and the jack. Positioning the camera in the ceiling, directly above the court could be one possible solution for this issue. However, from a general point of view, the results from the performed system test were encouraging.

For future reference, the used camera should be replaced by a different model, with a broader FOV $\left(>55^{\circ}\right)$ in order to cover all of the court's area. Furthermore, it is inconvenient to manually adjust the algorithm parameters, which depend of the surrounding environment, to acquire better results. Future work should also focus on developing an automatic calibration method for these parameters in order to make the system more easily adaptable to scenarios with different lighting conditions. Regarding future work, it is planned to test the proposed system, in real-time, during a Boccia game played by the elders living in the nursing home. It is also important to evaluate the usability of the system, which includes not only its effectiveness and efficiency, but also the resulting users' satisfaction towards the use of the system to understand if it can be a factor that influences participation in the game.

Acknowledgements. This article is a result of the project Deus ex Machina: NORTE01-0145-FEDER-000026, supported by Norte Portugal Regional Operational Programme (NORTE 2020), under the PORTUGAL 2020 Partnership Agreement, through the European Regional Development Fund (ERDF).

\section{References}

1. W.H.O., "Global recommendations on physical activity for health", Geneva: World Health Organization, 2010.

2. Lee, I.-M.; Shiroma, E.J.; Lobelo, F.; Puska, P.; Blair, S.N. and Katzmarzyk, P.T., "Impact of Physical Inactivity on the World's Major Non-Communicable Diseases", The Lancet, vol. 380, 219-29, 2012.

3. Matthews, C.E.; Chen, K.Y.; Freedson, P.S.; Buchowski, M.S.; Beech, B.M.; Pate, R.R. et al., "Amount of time spent in sedentary behaviors in the United States, 2003-2004", American Journal of Epidemiology, vol. 167, 875-81, 2008. 
4. Stessman, J.; Hammerman-Rozenberg, R.; Cohen, A.; Ein-Mor, E.; Jacobs, J.M., "Physical activity, function, and longevity among the very old", vol. 169, 1476-83, 2009.

5. Gillespie, L.D.; Robertson, M.C.; Gillespie, W.J.; Sherrington, C.; Gates, S.; Clemson, L.M. et al., "Interventions for preventing falls in older people living in the community", Cochrane Database of Systematic Reviews, 2012.

6. Westhoff, T.H.; Franke, N.; Schmidt, S.; Vallbracht-Israng, K.; Meissner, R.; Yildirim, H. et al., "Too old to benefit from sports? The cardiovascular effects of exercise training in elderly subjects treated for isolated systolic hypertension", Kidney and Blood Pressure Research, vol. 30, 240-7, 2007.

7. Salguero, A.; Martínez-García, R.; Molinero, O. and Márquez, S., "Physical activity, quality of life and symptoms of depression in community-dwelling and institutionalized older adults", Archives of Gerontology and Geriatrics, vol. 53, 152-7, 2011.

8. Schutzer, K.A. and Graves, B.S., "Barriers and motivations to exercise in older adults", Preventive Medicine, vol. 39, 1056-61, 2004.

9. Silva, V.; Ramos, J.; Soares, F.; Novais, P.; Arezes, P.; Figueira, C. et al., "A wearable and non-wearable approach for gesture recognition - Initial results", The 9Th International Congress on Ultra Modern Telecommunications and Control Systems, 185-90, 2017.

10. Figueira, C.; Silva, J.; Santos, A.; Sousa, F.; Silva, V.; Ramos, J. et al., "iBoccia: Monitoring elderly while playing Boccia gameplay", ICINCO 2017, vol. 1, 670-5, 2017.

11. Pingali, G.; Opalach, A. and Jean, Y., "Ball tracking and virtual replays for innovative tennis broadcasts", Proceedings-International Conference on Pattern Recognition, vol. 15, 152-6, 2000.

12. Wu, L.; Meng, X.; Liu, X. and Chen, S., "A new method of object segmentation in the basketball videos", Proceedings - International Conference on Pattern Recognition, vol. 1, 319-22, 2006.

13. Chen, H.T.; Tien, M.C.; Chen, Y.W.; Tsai, W.J. and Lee, S.Y., "Physics-based ball tracking and $3 \mathrm{D}$ trajectory reconstruction with applications to shooting location estimation in basketball video", Journal of Visual Communication and Image Representation, Elsevier Inc. vol. 20, 204-16, 2009.

14. Chen, H.T.; Tsai, W.J.; Lee, S.Y. and Yu, J.Y., "Ball tracking and 3D trajectory approximation with applications to tactics analysis from single-camera volleyball sequences", Multimedia Tools and Applications, 1-27, 2011.

15. Kim, J., "Curling Stone Tracking by an Algorithm Using Appearance and Colour Features", Proceedings of the World Congress on Electrical Engineering and Computer Systems and Science (EECSS), 1-6, 2015.

16. Kalman, R.E., "A New Approach to Linear Filtering and Prediction Problems 1", Journal of Fluids Engineering, vol. 82, 35-45, 1960.

17. Wang, J. R.; Parameswaran, N., "Survey of Sports Video Analysis: Research Issues and Applications", School of Compute Science and Engineering The University of New South Wales, vol. 113, 115-8, 2006.

18. BISFed, "BISFed International Boccia Rules (v.2)", 2017.

19. Silva, V.; Ramos, J.; Soares, F.; Novais, P.; Arezes, P.; Sousa, F., Silva, Jo; Santos, A., "iBoccia: A Framework to Monitor the Boccia Gameplay in Elderly", Lecture Notes in Computational Vision and Biomechanics, vol. 27, 2018. 